\title{
Developments of Finite-Volume Techniques for Electromagnetic Modeling in Unstructured Meshes
}

\author{
Christophe Fumeaux ${ }^{1}$, Dirk Baumann ${ }^{1}$, Pierre Bonnet ${ }^{2}$, Rüdiger Vahldieck ${ }^{1}$ \\ ${ }^{1}$ Swiss Federal Institute of Technology (ETHZ), IFH, Zürich, Switzerland, fumeaux/dbaumann/vahldieck@ifh.ee.ethz.ch \\ ${ }^{2}$ Université Blaise Pascal, LASMEA, Clermont-Ferrand, France, pierre.bonnet@lasmea.univ-bpclermont.fr
}

\begin{abstract}
This paper provides an overview of the recent developments of finite-volume techniques for time-domain electromagnetic simulations. The distinctive characteristic of Finite-Volume Time-Domain (FVTD) algorithms is their applicability in non-Cartesian volume meshes. This allows great geometrical flexibility which is required for most EMC problems. Several aspects of the FVTD method are reviewed, its applications to EMC problems are discussed and illustrated by simulation examples.
\end{abstract}

\section{INTRODUCTION}

Numerical analysis tools for electromagnetic fields have become an indispensable tool in the design of antennas and microwave circuits. The rapid progress in computer technology has allowed a steady increase in the size and complexity of the structures that can be modeled. In parallel, there has been ongoing research focused on the development of sophisticated computational methods with enhanced performance. The coexistence of diverse numerical techniques is essential since no single method can claim an overall superiority (in efficiency and accuracy) for all standard and non-standard problems encountered in the multitude of today's electromagnetic systems. Considering general field solvers, great efforts are made today towards the improvement of the most prominent methods like the Finite-Difference Time-Domain method (FDTD), the Finite-Element Method (FEM), the Transmission Line Method (TLM) or the various Method of Moments (MoM) approaches. Besides advances in this stream of research, less known methods are also being investigated, such as the class of finite-volume techniques.

The Finite-Volume Time-Domain (FVTD) method has been introduced for electromagnetic applications at the very beginning of the 90's [1],[2]. The FVTD method can be considered in-between FDTD and the FEM applied in time domain. The most essential characteristic of FVTD is its applicability in unstructured meshes. This gives the method a high geometrical flexibility and a versatility that is best exploited for complex problems with curved surfaces, for devices with multiscale structures in close proximity, or for the treatment of high dielectric constant materials and their interface to other media.

Since the FVTD method is still in its infancy, its potential has not been completely unfolded yet. The goal of this paper is to provide a snapshot of the present development of FVTD in view of its application to realworld electromagnetic problems. To begin with, Sect. II gives a short theoretical description of the method fundamentals. The third section deals with the implementation of the algorithm, addressing the space discretization and the treatment of boundaries, the time discretization, and the hybridization with other methods.
Other aspects relevant to the simulation of microwave devices and systems are also described, such as the extraction of generalized S-parameters, and the modeling of thin wires. The fourth section presents two examples of computation, a log-periodic antenna with a finely resolved feed structure, and a choked circular waveguide. The numerical results are compared with reference data, showing excellent agreement. The conclusion provides an outlook which aspects of the FVTD need to be addressed in the future to increase the applicability of the method.

\section{The Finite-Volume Time-Domain MethoD}

The FVTD method is based on Maxwell's curl equations written in their conservative form [3]. Integrating the equations over a finite volume $V$ leads to the volumesurface integrals

$$
\begin{aligned}
-\frac{\partial}{\partial t} \iiint_{V} \vec{B} d v & =\oiint_{\partial V} \vec{n} \times \vec{E} d a \\
\frac{\partial}{\partial t} \iiint_{V} \vec{D} d v & =\oiint_{\partial V} \vec{n} \times \vec{H} d a
\end{aligned}
$$

where $\partial V$ represents the boundary enclosing $V$ and $\vec{n}$ the outward-pointing normal unit vector of the infinitesimal surface element $d a$. In analogy to corresponding fluid dynamics conservation equations, the arguments in the right-hand side (RHS) surface integrals of (1) represent fluxes through the boundary of the volume.

For numerical implementation, the computational volume is partitioned in small polyhedral cells with volumes $V_{i}$. Assuming constant permittivity $\varepsilon_{i}$ and permeability $\mu_{i}$ in each elementary cell, the continuous equations (1) are discretized to

$$
\begin{aligned}
-\mu_{i} \frac{\partial}{\partial t}\langle\vec{H}\rangle_{V_{i}} & =\frac{1}{V_{i}} \sum_{k=1}^{N_{i}}\left(\vec{n}_{k} \times\langle\vec{E}\rangle_{F_{k}}\right) F_{k} \\
\varepsilon_{i} \frac{\partial}{\partial t}\langle\vec{E}\rangle_{V_{i}} & =\frac{1}{V_{i}} \sum_{k=1}^{N_{i}}\left(\vec{n}_{k} \times\langle\vec{H}\rangle_{F_{k}}\right) F_{k} .
\end{aligned}
$$

The sum on the RHS of (2) is performed for the $i$ th cell over its $N_{i}$ polygonal faces with areas $F_{k}$ and normal vector $\vec{n}_{k}\left(k=1, \ldots, N_{i}\right)$. The triangular brackets indicate spatially averaged field values over volumes (left-hand side, LHS) or face areas (RHS).

The FVTD method is characterized by a large number of algorithm variations. The present paper considers a representative FVTD structure with a cell-centered, collocated formulation: Both the electric and magnetic fields are sampled in the barycenters of the elementary cells. Furthermore, most implementations are based on characteristics theory [2] and consider a flux-splitting 
treatment, which separates outgoing $(+)$ and incoming (-) fluxes at each interface between two cells [3]

$$
\begin{aligned}
& \mu \vec{\Phi}^{E}=\vec{n}_{k} \times\langle\vec{E}\rangle_{F_{k}}=\vec{n}_{k} \times\langle\vec{E}\rangle_{F_{k}}^{+}-\vec{n}_{k} \times\langle\vec{E}\rangle_{F_{k}}^{-} \\
& \varepsilon \vec{\Phi}^{H}=\vec{n}_{k} \times\langle\vec{H}\rangle_{F_{k}}=\vec{n}_{k} \times\langle\vec{H}\rangle_{F_{k}}^{+}-\vec{n}_{k} \times\langle\vec{H}\rangle_{F_{k}}^{-} .
\end{aligned}
$$

Among alternative algorithms, the flux average formulation of [4] and the staggered formulation of [1] are representative of possible variations.

\section{PRACTICAL FVTD IMPLEMENTATIONS}

The practical aspects of FVTD implementations presented here stems mostly from [3],[5]-[7]. Most of the concepts are however adaptable in the general frame of finite-volume techniques.

\section{A. Space discretization, boundary conditions}

The volume integration of the conservative form of Maxwell's curl equations (2) permits application in any polyhedral mesh. Most common practical meshes are made of tetrahedrons, hexahedrons or prisms. In particular, tetrahedral meshes are relatively easy to handle and permit a very accurate approximation of complex boundaries. Large inhomogeneities of cell sizes can be achieved over a relatively short distance, which proves advantageous in multiscale problems. However, a tradeoff between rapid cell size increase and high accuracy is required since strongly distorted meshes result in a decrease of the overall solution accuracy.

Cell-centered finite-volume implementations permit a natural treatment of boundaries. The continuity equations for the tangential electric fields at material interfaces are satisfied, by taking into account the appropriate material properties in the computation of the incoming and outgoing fluxes [2]. The same applies to the boundary condition at the surface of conductors.

For radiation problems, absorbing boundary conditions (ABC) are necessary to terminate the computational domain. The Silver-Müller ABC is a simple boundary condition that sets the incoming FVTD fluxes to zero at the outer domain boundary. Although first order in nature (i.e. exact for normal incidence), the Silver-Müller ABC can provide an efficient suppression of reflections in practical cases: Using a spherical outer boundary for radiation problems provides a near-normal incidence condition at the outer boundary, which results in typical reflection coefficients lower than $-40 \mathrm{~dB}$.

\section{B. Time discretization, stability, local time-stepping}

A discrete approximation of the time derivative in the LHS of (2) allows finding explicit equations for the marchin-time iteration of the FVTD algorithm. A straightforward finite difference approximation corresponds to the Euler scheme, which benefits from its simplicity but is only first order in accuracy. For increased stability and accuracy, higher-order schemes such as the Lax-Wendroff predictorcorrector scheme or the Runge-Kutta method are advantageously used [3]. For any of the explicit forms, stability of the computation is achieved if the CourantFriedrich-Lévy (CFL) criterion is fulfilled. In an unstructured mesh however, all the cells are different and the "worst" cell in the mesh sets the maximum time step for stability. A commonly accepted criterion for the spatial order one considers the minimum volume/surface ratio of the cells over the whole mesh according to

$$
d t \leq \frac{1}{c} \min _{i}\left(\frac{\left|V_{i}\right|}{\sum_{k=1}^{N_{i}}\left|F_{k}\right|}\right)
$$

According to this criterion, it becomes clear that a highquality mesh avoids strongly irregular cells (i.e. needleshaped or flat tetrahedrons). Assuming an inhomogeneous mesh of good quality, the smallest cell determines then the time step for stability. If extremely small cells are necessary for resolving structural details, the length of the time step might be reduced dramatically.

To address this problem, a time-stepping scheme has been introduced in [6] which permits to save computer resources by adapting the duration of the time step locally to match the spatial discretization. The separation in subdomains with different time steps is performed automatically on the basis of the geometrical and material characteristics of the mesh. Special care has to be taken at the boundary between domains to avoid numerical reflection caused by time inconsistencies. Using an increment (or decrement) by a factor of two in (local) time steps between adjacent sub-domains permits to limit unphysical reflection to under $-50 \mathrm{~dB}$ while increasing strongly the efficiency of the computation.

Pushing the idea further, the use of different timestepping schemes in different areas of the computational domain might lead to additional increase in efficiency. For example, an implicit unconditionally stable time-stepping scheme could be applied advantageously in the subdomains with the smallest cells, trading the stability condition (4) for an accuracy-based criterion.

The multi-domain approach exploits the advantage of various time-stepping schemes (remaining in the frame of FVTD) where they perform best. This principle can be extended to different numerical methods in so-called hybrid approaches.

\section{Hybridization}

Hybridization of FVTD with other methods represents an attractive strategy to improve the CPU time and memory requirements. Along this line, examples have been published combining finite volumes and finite differences, which merge easily (e.g. [3],[8]).

FVTD and FDTD are local methods for which the evaluation of the fields in each cell only requires the value of the fields located in the surrounding cells. Consequently, the hybridization is performed by defining a transition area between the two schemes, in which field values are exchanged. In practice, FVTD with an unstructured conformal mesh is used to describe conformally the scatterer and its immediate neighbourhood with relatively few cells, whereas FDTD on a Cartesian grid is used for the remaining part of the computational domain. The transition area is composed of hexahedral cells (Fig. 1). The hybridization process is carried out using one or more overlapping cell layers where the boundary fields 
for each scheme are interpolated in space and time from fields calculated with the other method.

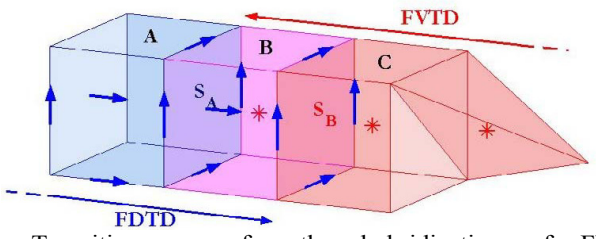

Fig. 1. Transition area for the hybridization of FV/FDTD. Interpolation of the electrical field given by FDTD (respectively FVTD) is performed on surface $S_{B}$ (respectively $S_{A}$ ).

This hybridization method keeps on one hand the efficiency of the FDTD in the major part of the computational domain and permits the use of efficient ABCs (as PML) developed for this scheme. On the other hand, FVTD provides the conformity of the studied objects which is fundamental for modelling wires or resonant structures. Nevertheless, considering hexahedral cells, the CFL number for the FVTD scheme (4) is more restrictive than for the FDTD method. This difference in time steps is reinforced by the fact that tetrahedral cells are generally smaller than the cubic cells. Therefore, to improve the efficiency of the hybridization in terms of CPU time, a different time stepping can be introduced for the two domains according to their CFL condition. The two domains are linked by performing adequate time interpolation at their interface. Figure 2 shows an example of a hybrid mesh.

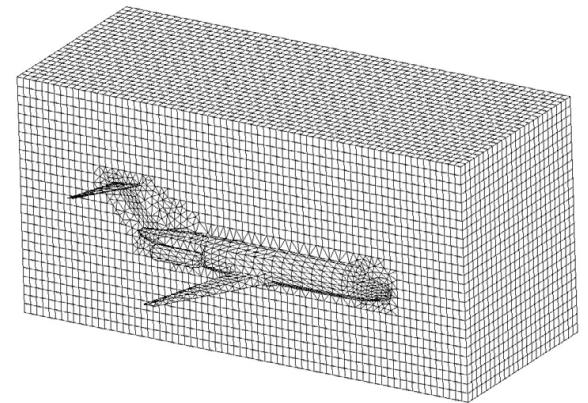

Fig. 2. Hybrid FV/FDTD mesh showing the structured and unstructured domains.

\section{Application to devices: S-parameter extraction}

Scattering parameters are a very important tool to evaluate microwave devices, and thus the accuracy of their extraction in a numerical simulation is crucial. To be able to extract a generalized scattering matrix, a port plane, defining a phase reference for the incident and the reflected wave, has to be introduced, either as a meshed surface inside the FVTD grid, or as an imaginary plane grid of interpolation points.

In the port plane, the outgoing $\vec{\Phi}^{+}$and the incoming $\vec{\Phi}^{-}$flux, inherently computed in the frame of the FVTD algorithm, are connected to the incident and the reflected wave in the simulated device [9]. The tangential fields in the port, required for the S-parameter extraction, are retrieved from the separated fluxes through

$$
\vec{E}_{t}^{+/-}=-\vec{n} \times \mu \vec{\Phi}^{E^{+/-}} .
$$

Once the tangential fields are known, the mode amplitude $A^{+/-}$of the considered mode can be extracted, provided that the mode template vector $\vec{e}$ and $\vec{h}$ are known and adequately normalized

$$
A^{+/-}=\sum_{k=1}^{K}\left(\vec{E}_{t}^{+/-}(k) \times \vec{h}(k)\right) F_{k},
$$

where $F_{k}$ is the area of the $k$ th face of the port plane consisting of $K$ triangles. Knowing the mode amplitudes, the generalized scattering matrix can be extracted.

In non-TEM structures, a correction factor has to be introduced for computation of the tangential fields in (5) to assure the correct power flow in the port plane [9]. An example for the extraction of the scattering parameters of a waveguide discontinuity is depicted in Fig. 3.

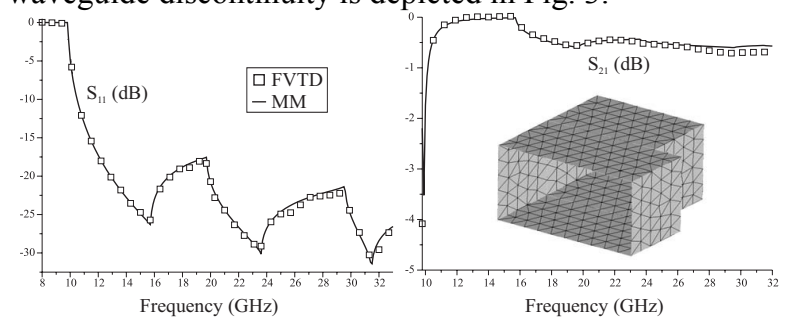

Fig. 3. Scattering parameters of a waveguide discontinuity. The FVTD simulation is benchmarked with Mode Matching (MM) results.

\section{E. Application to systems: wire/cable models}

In EMC as well as in studies of antennas, it is often advantageous to introduce a specific technique to model thin wires. In the FDTD method, thin-wire formalism was proposed by R. Holland [10]. This formalism is used for wires of very small diameter compared to the mesh size. In standard FDTD, wires are generally parallel to the cell axis and when this is not the case, the staircase approximation modifies the length of the wire and hence its resonance frequencies. This formalism has been extended to FVTD [5]. As in FDTD, the volume of the wire then does not require to be meshed. The wire is split into several segments in the middle of which current $I$ and charges $q$ are estimated at the same times. Then in a similar way as for the spatial fields, the wire equation (without source) can be cast into a conservative form as follows

$$
\frac{\partial \omega}{\partial t}+A \frac{\partial \omega}{\partial l}=0 \text { where } \omega=\left(\begin{array}{l}
I \\
q
\end{array}\right) \text { and } A=\left(\begin{array}{ll}
0 & c^{2} \\
1 & 0
\end{array}\right) \text {. }
$$

Therefore a finite volume discretization can be applied to (7). The coupling between space and wire is obtained by the tangential electric field around each wire segment. In the discretized FVTD domain, wires run along the edges of the elementary cells. In order to ensure good coupling, prism-shaped cells are used around the wires as illustrated in Fig. 4.

This thin wire formalism has been successfully applied in FVTD [3] and it is likely that other formalisms like multi-wire or thin slots will be developed in the future.

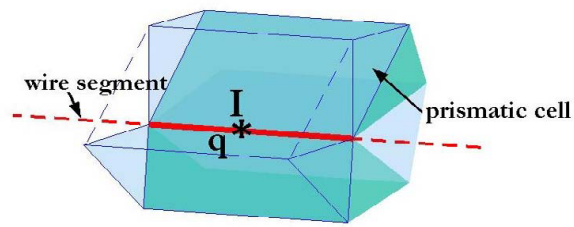

Fig. 4. Detail of the specific mesh around a wire segment. 


\section{APPLICATION EXAMPLES}

Traditional applications of the FVTD method have considered scattering problems for the determination of radar cross-section (e.g., [2],[5],[11]). In the present paper, two examples of antennas are presented.

\section{A. Log-periodic antenna}

The first example considers a trapezoidal-tooth logperiodic antenna for 1-12 GHz shown in Fig. 5. The use of the unstructured tetrahedral mesh is advantageous to resolve the oblique arms of the antenna, the thickness of the metal and dielectric parts as well as the fine coaxial feeding structure. The results of the simulation show a very good agreement with measured data over the full frequency range. As illustration, radiation patterns are shown in Fig. 6 for two sample frequencies.

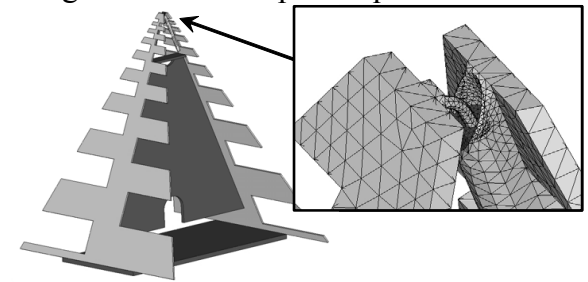

Fig. 5. Trapezoidal-tooth log-periodic antenna for 1-12 GHz. The light gray parts represent the metallic arms and the darker gray parts dielectric plates that provide mechanical stability. The inset shows a magnified view of the triangulated feed.

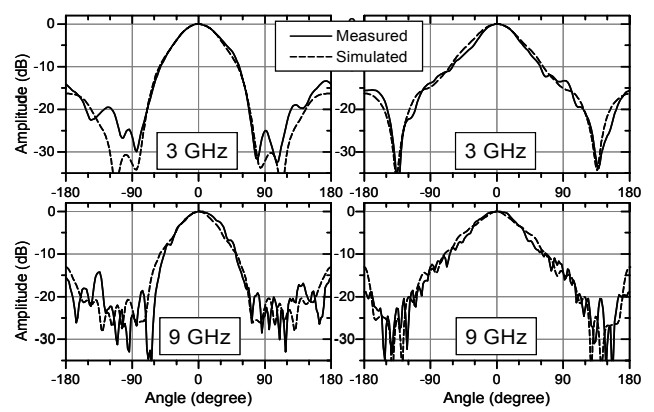

Fig. 6. Comparison of simulated and measured patterns for the logperiodic antenna at two frequencies. LHS: E-plane, RHS: H-plane.

\section{B. Choked waveguide}

For applications where low cross-polarization is required, multi-mode horns are a common choice. As an example, a choked waveguide, mostly employed for illumination of reflectors, has been simulated. The circularly symmetric pattern is a consequence of appropriate superposition of the $\mathrm{TE}_{11}$ mode in the waveguide aperture and the coaxial $\mathrm{TE}_{11}$ mode excited in the choke. Co- and cross polarizations and the return loss simulated with FVTD are compared to a Mode-Matching (MM) reference solution in Fig. 7.

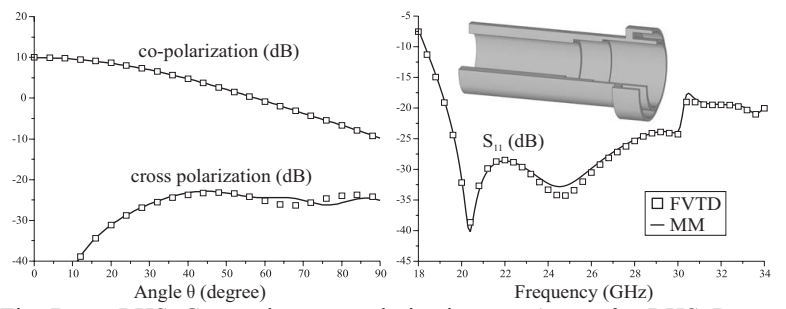

Fig. 7. LHS: Co- and cross polarization at $\phi=45^{\circ}$; RHS: Return loss of the choked waveguide. The FVTD simulation is benchmarked with Mode Matching (MM) results.

\section{FUTURE DEVELOPMENTS AND CONCLUSION}

This paper has reviewed recent developments of the FVTD method mainly in view of its use for EMC problems. The main advantage of the method consists in its use of an unstructured mesh. This allows the resolution of arbitrarily-shaped structures for complex electromagnetic problems.

The FVTD method, as a relatively new method, still has potential for improvement. Among the many different variations of finite-volume algorithms, efforts are directed towards finding the algorithms exhibiting the best spatial convergence of the solutions, i.e. allowing the use of larger elementary cells. This might also be achieved using higher order algorithms (e.g. discontinuous Galerkin method).

Although the FVTD method allows to reduce the number of cells necessary to mesh complex structures, the memory and CPU requirements per cell remain high in comparison with FDTD. Therefore, efforts are also required towards the development of acceleration and hybridization techniques. Model Order Reduction (MOR) in conjunction with finite-volume techniques [12] represents also a promising track of exploration.

\section{REFERENCES}

[1] N.K. Madsen, R.W. Ziolkowski, "A three-dimensional modified finite volume technique for Maxwell's equations", Electromagnetics, vol. 10, pp. 147-161, 1990.

[2] V. Shankar, A.H. Mohammadian, W.F. Hall, "A time-domain, finite-volume treatment for the Maxwell equations", Electromagnetics, vol. 10, pp. 127-145, 1990.

[3] P. Bonnet, X. Ferrières, B. Michielsen, and P. Klotz, Time Domain Electromagnetics. S. M. Rao, Ed., Academic Press, 1997, ch. 9, pp. 307-367.

[4] S. Piperno, M. Remaki, L. Fezoui, "A nondiffusive finite volume scheme for the three-dimensional Maxwell's equations on unstructured meshes", SIAM J. Numer. Anal., vol. 39, no. 6, pp. 2089-2108, 2001.

[5] P. Bonnet, X. Ferrières, F. Issac, F. Paladian, J. Grando, J.C. Alliot, J. Fontaine, "Numerical modeling of scattering problems using a time domain finite volume method", J. of Electromagnetic Waves and Appl., vol. 11, pp. 1165-1189, 1997.

[6] C. Fumeaux, D. Baumann, P. Leuchtmann, R. Vahldieck, "A generalized local time-step scheme for efficient FVTD simulations in strongly inhomogeneous meshes", IEEE Trans. Microwave Theory Tech. MTT-52(3), pp. 1067-1076, 2004.

[7] D. Baumann, C. Fumeaux, P. Leuchtmann, R. Vahldieck, "Finitevolume time-domain (FVTD) modeling of a broadband doubleridged horn antenna", Int. J. Numer. Model., vol. 17, no. 3, pp. 285298, 2004.

[8] M. Yang, Y. Chen, R. Mittra, "Hybrid finite-difference/finitevolume time-domain analysis for microwave integrated circuits with curved PEC surfaces using a nonuniform rectangular grid", IEEE Trans Microwave Theory Tech., vol 48, no. 6, pp.969-975, June 2000.

[9] D. Baumann, C. Fumeaux, P. Leuchtmann, R. Vahldieck, "Fieldbased scattering-matrix extraction scheme for the FVTD method exploiting a flux-splitting algorithm", in print, IEEE Trans. Microwave Theory Tech., vol. 53, no. 11, 2005.

[10] P. Holland and L. Simpson, "Finite difference analysis of EMP coupling to thin struts and wires", IEEE Trans. on EMC, vol. 23, pp 88-97, May 1981.

[11] R. Holland, V.P. Cable, L.C. Wilson, "Finite-Volume Time-Domain (FVTD) techniques for EM scattering", IEEE Trans. on EMC, vol. 33, no. 4, pp 281-294, 1991.

[12] K. Krohne, D. Baumann, C. Fumeaux, R. Vahldieck, "Reduced order finite volume models", Proc. 34th European Microwave Conf., Amsterdam, pp. 581-584, Oct. 2004. 